\title{
Giant posterior pararenal schwannoma: A case report and review of literature
}

\author{
XIQI PENG ${ }^{1,2^{*}}$, ZUWEI LI $^{1,2^{*}}$, LIANG ZHOU ${ }^{1,3}$, LIWEN ZHAO $^{1}$, XIONGHUI WU ${ }^{1}$, \\ YU YANG ${ }^{1}$, SHANGQI YANG ${ }^{1}$, YUN CHEN $^{4}$ and YONGQING LAI ${ }^{1}$ \\ ${ }^{1}$ Department of Urology, Peking University Shenzhen Hospital, Shenzhen, Guangdong 518036; \\ ${ }^{2}$ Shantou University Medical College, Shantou, Guangdong 515041; ${ }^{3}$ Department of Urology, \\ Guangzhou Medical University, Guangzhou, Guangdong 510006; ${ }^{4}$ Department of Ultrasound, \\ Peking University Shenzhen Hospital, Shenzhen, Guangdong 518036, P.R. China
}

Received February 4, 2018; Accepted June 29, 2018

DOI: $10.3892 / \mathrm{mco} .2018 .1664$

\begin{abstract}
Schwannomas, arising from Schwann cells of peripheral nerve sheaths, are primarily benign tumors. They are rarely found in the retroperitoneal space. To date, $\sim 30$ cases on giant retroperitoneal schwannomas have been reported. Those with a location of pararenal space are even rarer. Clinically, they are often misdiagnosed as malignancies. In the present study, a case of a 35-year-old woman with a giant schwannoma measuring $13 \times 8.5 \times 6.5 \mathrm{~cm}$ in the posterior pararenal space of left retroperitoneum was presented, which was thought to be a malignant tumor from the result of computed tomography scan. Later postoperative pathology and immunohistochemistry were consistent with benign schwannoma. To the best of our knowledge, this was the largest posterior pararenal schwannoma reported in literature. It is significant to report this case, and summarize the characteristics, diagnosis, treatment and prognosis of the similar cases. Furthermore, a brief review of the previously published cases in literature is provided.
\end{abstract}

\section{Introduction}

Schwannomas are mostly benign tumors. They are often seen in the head, neck and extremities. Studies have shown that only 0.7 to $2.6 \%$ of schwannomas are found in the retroperitoneum.

Correspondence to: Professor Yongqing Lai, Department of Urology, Peking University Shenzhen Hospital, 1120 Lianhua Road, Shenzhen, Guangdong 518036, P.R. China

E-mail: yqlord@163.com

Professor Yun Chen, Department of Ultrasound, Peking University Shenzhen Hospital, 1120 Lianhua Road, Shenzhen, Guangdong 518036, P.R. China

E-mail: cyun126@126.com

${ }^{*}$ Contributed equally

Key words: giant, pararenal schwannoma, literature review, tumor, retroperitoneum
Those in pararenal space are even rarer. Although most schwannomas are $<5 \mathrm{~cm}$ in diameter, retroperitoneal schwannomas are able to grow into a large size in such a non-restrictive space (1). They are mostly asymptomatic until they grow too large to cause compression, which is usually ambiguous pain. In the present study we introduce a case of a 35-year-old female with a giant posterior pararenal schwannoma. To the best of our knowledge, this is the first time to report a posterior pararenal schwannoma in such a large size.

\section{Materials and methods}

The surgical specimen were fixed in $10 \%$ formalin at room temperature overnight and embedded in paraffin. Later, the specimen were cut into four-micrometer-thick sections. Before staining, the sections were dewaxed in xylene, rehydrated through decreasing concentrations of ethanol, and washed in PBS. After that, the sections were stained with hematoxylin at $37^{\circ} \mathrm{C}$ for $15 \mathrm{~min}$, rinsed under running water for $1 \mathrm{~min}$ and then left standing in water for $5 \mathrm{~min}$. Similarly, Staining with $0.5 \%$ eosin was executed for at $37^{\circ} \mathrm{C}$ for $3 \mathrm{~min}$, followed by rinsing under running water. After staining, sections were dehydrated through increasing concentrations of ethanol and xylene. Rehydration was performed by continuous immersion in xylene, graded concentrations of ethanol, and tap water. Slides were kept in citrate buffer $(\mathrm{pH} \mathrm{9.0)} \mathrm{and} \mathrm{two} \mathrm{cycles} \mathrm{of}$ heat retrieval were performed in oven at $99^{\circ} \mathrm{C}$ for ten and five minutes.

Slides were washed in Tris buffer ( $\mathrm{pH}$ 7.8). To blocking endogenous peroxidase, all sections were incubated with hydrogen peroxide for $10 \mathrm{~min}$. Next, sections were washed thrice in Tris buffer, followed by $30 \mathrm{~min}$ incubation with primary antibodies. Primary antibodies used were S100 protein (cat. no. PIPA516257; rabbit polyclonal antibody, dilution 1:300), CD34 (cat. no. PIMA516924; mouse monoclonal antibody; QBend/10; dilution 1:100), SMA (cat. no. PIMA511547; mouse monoclonal antibody; clone 1A4; dilution 1:200), and Ki-67 (mouse monoclonal antibody; clone 4A1) (all from Invitrogen, Waltham, MA, USA). Secondary antibody including goat anti-mouse IgG (cat. no. PIA32723; dilution 1:500) and goat 


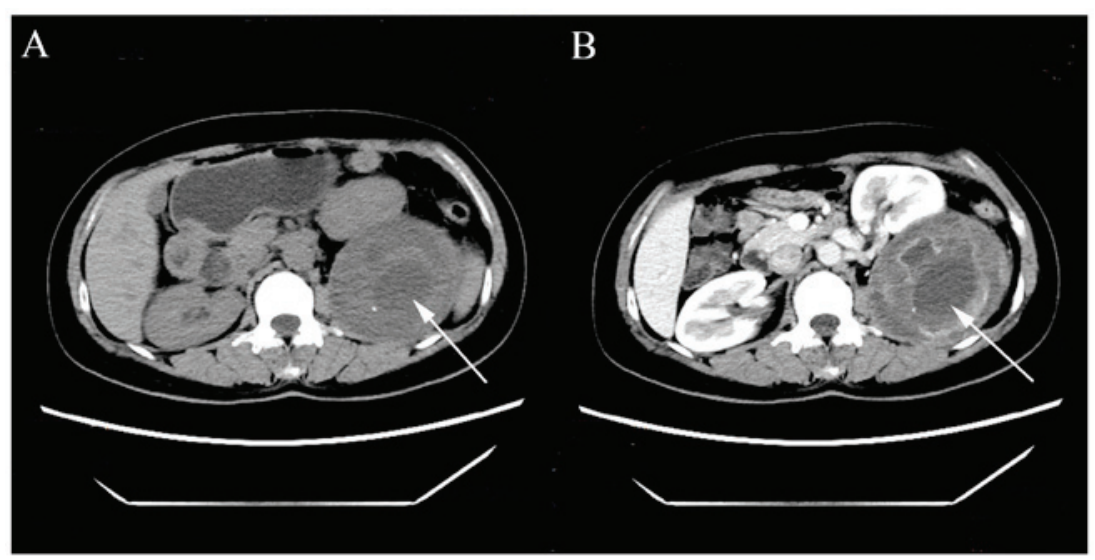

Figure 1. (A) Non-enhanced axial computed tomography image revealing a 89 × 81 x $104 \mathrm{~mm}, 13-48 \mathrm{HU}$, well-demarcated, cystic solid mass with mixed density in the left posterior pararenal space (arrows), adhering with adjacent organs. The left kidney was pushed to inferior. (B) Contrast-enhanced axial computerized tomography image showing apparent heterogeneous contrast enhancement inside the mass. Arrows indicate the location of the giant schwannoma.

anti-rabbit IgG (cat. no. A11034; dilution 1:500) (both from Invitrogen) was added correspondingly after washing with Tris buffer for $40 \mathrm{~min}$. At last, chromogen diaminobenzidine (DAB) was added for $10 \mathrm{~min}$, followed by counterstaining with hematoxylin for $2 \mathrm{~min}$, sequential immersions in xylene and alcohol and mounting with distyrene plastisizer xylene (DPX).The results were observed under light microscope.

\section{Case report}

A 35-year-old female was admitted to Peking University Shenzhen Hospital (Shenzen, China) with an incidental finding of a left retroperitoneal mass 1 month earlier. Except for dull pain in left waist, no other symptoms showed up. On physical examination, a large, firm, smooth, non-tender mass was palpable. Ultrasound examination revealed a predominant solid mass measuring $109 \times 89 \times 86 \mathrm{~mm}$ between the left kidney and spleen, with multiple oval dark areas. Small amount of blood flow could be seen. CT scan revealed a giant cystic solid mass with mixed density in the left posterior pararenal space, adhering with pancreas, spleen, kidney and psoas major muscle. The left kidney was pushed to inferior with vascular circuity. The lesion was predominantly low density, 13-48 HU, well-demarcated, smooth with capsule, and measured $89 \times 81 \times 104 \mathrm{~mm}$ with punctiform calcification inside it. On enhanced CT, apparent heterogeneous contrast enhancement could be seen inside the mass. The left kidney and adrenal gland had a normal morphology and uniform density, showing no abnormality on enhanced CT. No swollen lymph nodes were found (Fig. 1). According to the results above, adrenal neoplasms needed to be distinguished, including pheochromocytoma, cortical adenoma, and aldosteronoma. The blood pressure of the patient was in normal range. Later hematologic examination showed that the secretion of ACTH and cortisol (at $\mathrm{OMN}, 8$ a.m. and 4 p. m.) had a normal circadian rhythm and was within normal range. Catecholamine, methoxy adrenalin, quantitative test of $24 \mathrm{~h} \mathrm{VMA}$ (vanilmandelic acid) were within normal limits. Angiotensin, renin, ALD (aldosterone) in blood showed no abnormalities in both erect position and clinostatism. Basically, adrenal neoplasms could be ruled out. However, a preoperative diagnosis was still challenging.

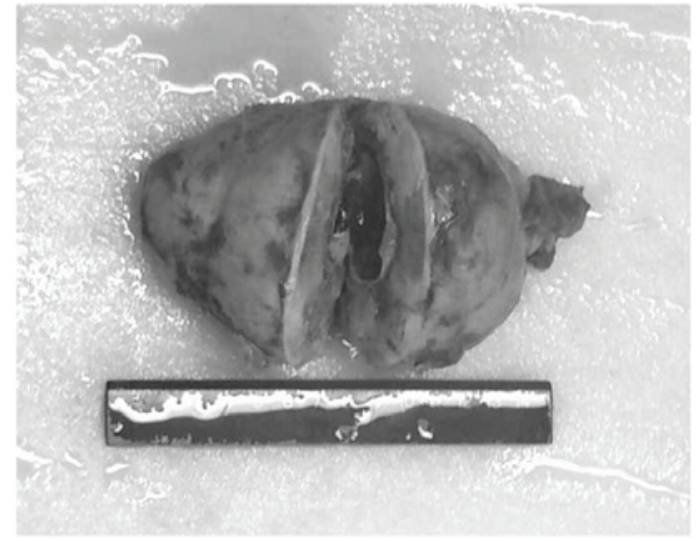

Figure 2. Gross examination showing a cystic solid mass measured $13 \times 8.5 \times 6.5 \mathrm{~cm}$ with intact smooth capsule.

Later, the patient underwent surgery. Intraoperatively, a cystic solid oval mass was found behind the kidney in the left retroperitoneum, with obvious adhesion with adjacent tissue. No obvious change in blood pressure of the patient was seen when touching the mass. The mass has an intact capsule and a complete resection was executed.

Gross examination showed a solid mass measuring $13 \times 8.5 \times 6.5 \mathrm{~cm}$, with cystic degeneration $(4.0 \mathrm{~cm}$ in diameter). It was wrapped with an intact smooth capsule (Fig. 2). On microscopy the mass was composed of spindle cells, with regions of high and low cellularity (Antoni A and B areas, respectively). Hemorrhagic and cystic degeneration could be seen in central area (Fig. 3).

Immonohistochemical staining revealed positivity for S100 and negative for CD34 and SMA. The Ki-67 proliferation index was about $10 \%$. Based on these, a diagnosis of schwannoma was suggested. During the follow-up, there was no evidence of recurrence.

\section{Discussion}

Schwannomas, arising from Schwann cells of peripheral nerve sheaths, are mostly benign tumors. They are often found in 


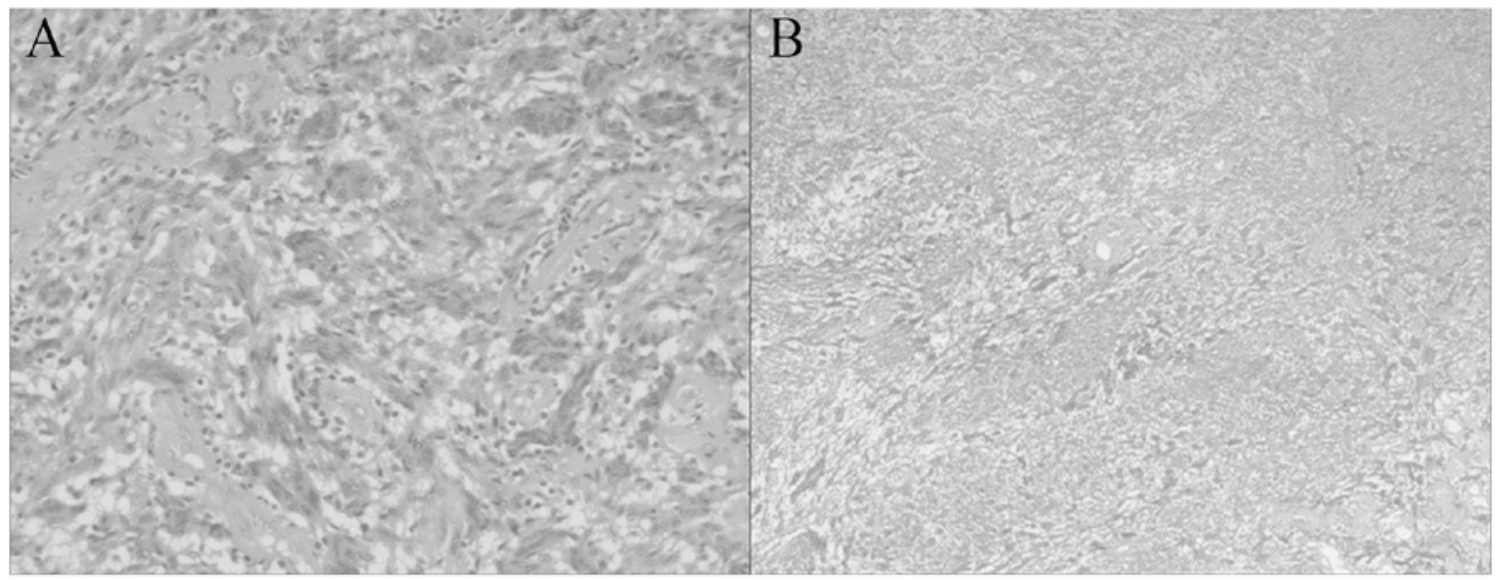

Figure 3. On microscopy the mass was composed of spindle cells, with regions of (A) high and (B) low cellularity. Hemorrhagic and cystic degeneration could be seen in central area. Magnification, x200.

the head, neck and extremities in the 4th and 6th decades of life (2) Studies have shown that only $0.7-2.6 \%$ of schwannomas are found in the retroperitoneum. Generally, schwannomas are less than $5 \mathrm{~cm}$ in diameter. But retroperitoneum is a nonrestrictive space which allows the tumors to reach a large size over a long time of growth (1). According to our search on Pubmed, we found out about 30 cases on giant retroperitoneal schwannoma. Yet, there have never reported a posterior pararenal schwannoma larger than $10 \mathrm{~cm}$ in diameter.

According to a literature published by G. Lannaci in 2016, twenty-one cases of primary renal schwannoma have been reported in literature (3). Schwannomas originating in the pararenal space are extremely rare. To date, only a few cases have been reported. Miyagi T reported a posterior pararenal schwannoma with a diameter of $3.0 \times 2.5 \mathrm{~cm}$ in 1986 (4). In 2010, a patient suffering from Neurofibromatosis Type 2 found a schwannoma posterior and inferior to the left kidney, measuring $9.5 \times 4 \times 4 \mathrm{~cm}$ (5). In 2013, Liu et al mentioned eight patients with retroperitoneal schwannomas in the anterior pararenal space (6). Here in, we show a giant posterior pararenal schwannoma measuring $13 \times 8.5 \times 6.5 \mathrm{~cm}$, which we believe to be the biggest one that has been reported in this location by far. This is the first time to report a pararenal schwannoma with a size more than $10 \mathrm{~cm}$ in diameter.

Schwannomas are mostly well-demarcated round or oval masses on CT and MRI. Cystic and hemorrhagic degeneration can be seen in large retroperitoneal schwannomas, which appear as inhomogeneous low-density masses and homogeneous to heterogeneous contrast enhancement on enhanced CT. MRI images can reminder the origin and the exact location of the mass. The intensity of the masses can help with the inference of their properties. However, these changes are non-specific. We are not able to make a final diagnosis through these radiographic results.

Blood biochemical examination is conducive to exclude adrenal neoplasms. The high concentration of certain hormones in blood can reveal the existence of functional adenoma. However, for non-functional adenoma, the hormones in blood could be normal, which means that blood biochemical examination only plays a limited role in diagnosis. Fine-needle aspiration biopsy is a way to get the pathological diagnosis of retroperitoneal masses. Yet it is suffered from controversy due to its low accuracy and potential complications. Still, preoperative diagnosis is challenging and a surgical removal is required for diagnosis.

For retroperitoneal masses larger than $4 \mathrm{~cm}$, especially those with clinical symptoms, surgical removal is regarded as the most rational way, with the benefit of both diagnosis and therapy. An incomplete excision may increase $5-10 \%$ of local recurrence (7). Moreover the possibility of malignancy is considerable. Therefore, a complete excision is necessary.

To make a final diagnosis, we need to combine pathology and immunohistochemistry. Histologically, there are two growth patterns in schwannomas. The Antony A pattern is characterized by a cell-rich structure with cell nuclei running parallel to each other while the Antony B pattern has a loose distribution of the cells with varying degrees of myxoid and hyaline degeneration. Immunohistochemically, S100 is an important marker for the diagnosis of schwannomas (8).

The prognosis of retroperitoneal schwannomas are mostly good. The most common complication is recurrence, possibly caused by incomplete excision. Malignant schwannomas has poor prognosis and a high rate of recurrence though the malignant transformation of retroperitoneal schwannomas appears to be extremely rare (9).

In the present study, we introduced a giant posterior pararenal schwannoma which was believed to be the largest one reported in this location by far. There is a dilemma on the preoperative diagnosis of pararenal schwannoma. It's important to include schwannomas during the differentiation of pararenal masses, especially for those with cystic and hemorrhagic degeneration since the treatment and prognosis among them may differ a lot.

\section{Acknowledgements}

Not applicable.

\section{Funding}

The present study was supported by the National Natural Science Foundation of China (grant no. 81101922), Science 
and Technology Development Fund Project of Shenzhen (grant nos. JCYJ20160429090753103 and JCYJ20170307111334308), Clinical Research Project of Shenzhen Health Commission (no. SZLY2018023), the fund of 'San-ming' Project of Medicine in Shenzhen (grant no. SZSM201612066), the Fund of Shenzhen Key Laboratory (grant no. ZDSYS201504301045406) and the fund of Guangdong Key Medical Subject.

\section{Availability of data and materials}

The datasets used during the current study are available from the corresponding author on reasonable request.

\section{Authors' contributions}

$\mathrm{XP}$ and ZL performed data collection, interpretation and drafted the manuscript. LiaZ and LiwZ contributed to the study design and acquisition of data. XW, YY, SY and YC participated in the study design, data collection, analysis of data and follow-up of the patient. YL contributed to the study design, and the analysis and interpretation of data.

\section{Ethics approval and consent to participate}

Written informed consent to participate was obtained from the patient.

\section{Patient consent for publication}

Consent for publication of any associated data and accompanying images was obtained from the patient.

\section{Competing interests}

The authors declare that they have no competing interests.

\section{References}

1. Karaköse O, Pülat H, Oğuz S, Zihni İ, Özçelik KÇ, Yalta TD and Eken H: A giant ancient schwannoma mimicking an adnexal mass: Case report. Medicine (Baltimore) 95: e4240, 2016.

2. Baten E, Lerut J and Kempeneers I: Hybrid open/closed resection procedure for ancient retroperitoneal Schwannoma: Case report and review of the literature. Acta Chir Belg 116: 289-292, 2016.

3. Iannaci G, Crispino M, Cifarelli P, Montella M, Panarese I, Ronchi A, Russo R, Tremiterra G, Luise R and Sapere P: Epithelioid angiosarcoma arising in schwannoma of the kidney: Report of the first case and review of the literature. World J Surg Oncol 14: 29, 2016.

4. Miyagi T, Shimamura M, Rin SY and Matsubara F: Retroperitoneal schwannoma: A report of two cases and review of the literature. Hinyokika Kiyo 32: 207-214, 1986.

5. Patrinou A, Malindretos P, Koutroubas G, Anagnostou N, Argiraki E and Syrganis C: A rare retroperitoneal schwannoma in a patient with neurofibromatosis Type 2. NDT Plus 3: 288-290, 2010.

6. Liu QY, Lin XF, Zhang WD, Li HG and Gao M: Retroperitoneal schwannomas in the anterior pararenal space: Dynamic enhanced multi-slice CT and MR findings. Abdom Imaging 38: 201-210, 2013.

7. Lee NJ, Hruban RH and Fishman EK: Abdominal schwannomas: Review of imaging findings and pathology. Abdom Radiol (NY) 42: 1864-1870, 2017.

8. Herden J, Drebber U, Ural Y, Zimmer S, Wille S and Engelmann UH: Retroperitoneal schwannomas of renal and pararenal origin: Presentation of two case reports. Rare Tumors 7: $5616,2015$.

9. Xu H, Sha N, Li HW, Bai M, Chen X, Hu HL and Wu CL: A giant pelvic malignant schwannoma: A case report and literature review. Int J Clin Exp Pathol 8: 15363-15368, 2015. 